\title{
O uso de métodos de previsão de demanda nas indústrias alimentícias brasileiras
}

\section{Demand forecasting methods in the brazilian food industries}

\author{
Cristiano Cecatto ${ }^{1}$ \\ Patrícia Belfiore ${ }^{2}$
}

\begin{abstract}
Resumo: O presente trabalho busca conhecer as práticas de previsão de demanda que vêm sendo utilizadas pelas indústrias de alimentos pesquisadas. Um questionário foi desenvolvido e enviado a uma amostra de 450 empresas do setor encontradas na ABIA (Associação Brasileira das Indústrias da Alimentação), que representa 70\% do universo, e um índice de resposta de 14,4\% foi alcançado. O objetivo da pesquisa é detectar quais e como os métodos de previsão vêm sendo utilizados por essas empresas, quais os principais fatores que influenciam na escolha do modelo e quais as principais dificuldades encontradas no uso de métodos de previsão de demanda. Os dados foram analisados por meio de técnicas estatísticas multivariadas, como análise de correspondência e análise discriminante, utilizando o software estatístico SPSS. Os resultados mostram que o modelo de análise histórica é o mais utilizado, que os principais fatores que influenciam a escolha do modelo são tipo de produto e tempo despendido, e que as maiores dificuldades são disponibilidade de softwares e dificuldade no entendimento.
\end{abstract}

Palavras-chave: Previsão de demanda; Análise multivariada; Análise de correspondência; Análise discriminante; Indústria de alimentos.

\begin{abstract}
This study aims to better understand the demand forecasting methods used by the Brazilian food industry. A questionnaire was developed and sent to a sample of 450 companies from ABIA (Brazilian Association of Food Industries) which represents $70 \%$ of the industry. Tthe response rate was $14.4 \%$. The goal of this research is to detect which and how the demand forecasting methods have been used by these companies, the main factors influencing the choice of method and what are the main obstacles that they face. The data was analyzed by multivariate statistical techniques such as correspondence analysis and discriminant analysis using the statistical software SPSS. The results show that the historical analysis model is the most used; that the main factors influencing the choice of models are the type of product and the time spent, and the main obstacles are the software availability and difficulty in understanding.
\end{abstract}

Keywords: Forecasting; Multivariate analysis; Correspondence analysis; Discriminant analysis; Food industry.

\section{Introdução}

O interesse em conhecer o desempenho das indústrias de alimentos no Brasil em termos de planejamento de demanda, instrumental necessário para se enfrentar mercados competitivos e em expansão, foi estimulado pela sua importância no cenário econômico e social do país. As deficiências que ainda podem ser apontadas no setor, no que tange a suprir o mercado e a adoção de inovações, além da escassez de dados em literatura com relação ao tema, contribuíram para o desenvolvimento desse trabalho.

Com relação à importância da indústria de alimentos, Birchal (2004) ressalta que, além de ser o setor responsável pelo primeiro impulso industrial brasileiro no final das últimas décadas do século 19, e representar, ao final da Primeira Grande Guerra, a segunda maior atividade no Brasil (superada somente pelas indústrias têxteis), sua importância se evidencia pelo conjunto dos dados agregados ao longo de todo o século 20.

O crescimento na indústria de alimentos resulta no aumento do volume de operações em agronegócios (produtores de matéria-prima), na distribuição atacadista e varejista, além das empresas de embalagens. Essas empresas representam hoje um segmento produtivo com significativa participação no crescimento econômico

${ }^{1}$ Departamento de Engenharia de Produção, Centro Universitário da FEI, Av. Humberto de Alencar Castelo Branco, 3972, Assunção, CEP 09850-901, São Bernardo do Campo, SP, Brasil, e -mail: cecatto@ qualilog.com

${ }^{2}$ Departamento de Engenharia de Gestão, Universidade Federal do ABC, Rua Rua Arcturus, 03 - Jardim Antares, CEP 09606-070, São Bernardo do Campo, SP, Brasil, e-mail: patricia.favero@ufabc.edu.br

Recebido em Maio 23, 2012 - Aceito em Abr. 02, 2015

Suporte financeiro: Nenhum. 
do Brasil, além de expressiva participação no Produto Interno Bruto (PIB).

Em 2007, segundo o Instituto Brasileiro de Geografia e Estatística, o Brasil tinha uma população estimada de 189,4 milhões de habitantes, com um PIB (preços de mercado) girando em torno de $\mathrm{R} \$ 2.634,1$ bilhões, sendo a produção da indústria em torno de R\$1.323,9 bilhões (IBGE, 2007). Nesse contexto, segundo a Associação Brasileira das Indústrias de Alimentação (ABIA, 2008), entidade de classe representativa do setor, a indústria de alimentos participa com $8,0 \%$ do PIB, um faturamento líquido de $\mathrm{R} \$ 230,6$ bilhões, cerca de 1.322,7 milhões de empregados no ano de 2007 e é responsável por $16,6 \%$ da exportação do país.

Apesar de números tão positivos, Conceição (2007) apresenta um estudo no qual identifica as características das empresas do setor de alimentos, destacando que ainda há muito a fazer para que possam melhorar seu desempenho. A própria ABIA aponta que em 2007 o crescimento do varejo se posicionou acima da produção industrial, e que a demanda foi suprida pelas importações. Conceição (2007) destaca, nesse estudo, que o percentual de adoção de inovações por esse segmento é muito pequeno.

Atualmente, empresas que mostram excelência no atendimento ao consumidor destacam-se também pelo planejamento de demanda, que possibilita direcionar os planos de produção, estoque, distribuição e compras, além de enfrentar questões mais complexas, como a proliferação de produtos, promoções e crescimento da concorrência. A relação da indústria com o consumidor ainda sofre mudanças, segundo a ABIA (2008), que destaca a necessidade de reconhecer as expectativas do cliente e estar atento à sua produção, para atender sua demanda.

O planejamento e a organização dos recursos produtivos possibilitam minimizar erros e atender à demanda dos consumidores. A tarefa é complexa, pois fatores não previstos ou que fogem ao domínio da empresa podem interferir. Para Birchal (2004), deve-se estar atento à importância estratégica da diferenciação de produtos e das economias de escala em produção. Porém, ainda é o método de planejamento de demanda, adequado e monitorado, que ajuda a empresa a atender à demanda de um mercado competitivo, minimizando erros e otimizando sua produção.

Nesse sentido, investimentos no planejamento de demanda podem trazer ganhos considerados para a organização (veja Taylor \& Fearne, 2009; Byrne et al., 2011). A escolha do modelo de previsão de demanda apropriado diante de situações específicas e similares possibilita às empresas direcionar seus planos de produção, estoque e compras, diminuir erros, suprir a demanda dos consumidores, além da definição de questões estratégicas mais complexas, tornando-se um fator crítico de sucesso para a gestão de negócios.

Jain \& Malehorn (2006) citam que não existe nenhum modelo de previsão "mágico" que sirva sempre e em todas as situações, e concluem que trabalhar com previsão é juntar ciência e arte.

\section{Objetivos e hipóteses}

O objetivo principal deste estudo é verificar qual o panorama atual das práticas de previsão de demanda nas indústrias de alimentos pesquisadas. Dessa forma, foram selecionadas 450 empresas do setor de alimentos encontradas na ABIA (Associação Brasileira das Indústrias da Alimentação). Os dados foram analisados através de estatística descritiva e técnicas estatísticas multivariadas.

Como objetivos específicos, o trabalho procura responder algumas questões e hipóteses que foram baseadas na literatura:

Jain \& Malehorn (2006) realizaram uma pesquisa junto às indústrias americanas de diferentes setores, para averiguar quais os modelos mais utilizados e que têm a preferência das indústrias. $\mathrm{O}$ resultado da pesquisa americana mostrou que as indústrias de alimentos, em sua quase totalidade, utilizam o modelo de alisamento exponencial para previsão de demanda. Pretende-se verificar se o mesmo também ocorre com as indústrias alimentícias pesquisadas na Questão 1.

Questão 1: As indústrias alimentícias pesquisadas utilizam algum método de previsão de demanda? Se sim, quais modelos estão sendo utilizados?

Hipótese 1: As indústrias alimentícias pesquisadas utilizam um dos modelos de previsão listados no questionário (Simulação, Pesquisa de mercado, Delphi, Painel de especialistas, Análise histórica, Média móvel, Alisamento exponencial, ARIMA, Regressão, Econométrico, Redes Neurais).

Hipótese 2: A maioria das empresas pesquisadas fornecedoras de produtos alimentícios não utiliza modelos sofisticados.

Hipótese 3: O modelo de previsão mais utilizado pelas indústrias alimentícias pesquisadas é o alisamento exponencial.

Hipótese 4: Não há consenso entre as indústrias de alimentos pesquisadas em relação à utilização de um único modelo de previsão de demanda.

A partir dos trabalhos de Winklhofer et al. (1996), Armstrong \& Fildes (2006), Küsters et al. (2006), Wilson \& Daubek (1989), Fildes \& Hastings (1994) e Naylor (1981), foram definidas as principais variáveis 
que podem influenciar na escolha de modelos de previsão de demanda. São elas: precisão, facilidade no entendimento, facilidade no uso, horizonte de previsão, custo, tempo despendido, consistência dos dados, disponibilidade de softwares para previsão, definindo a Questão 2.

Questão 2: Quais os principais fatores que influenciam a escolha do modelo?

Hipótese 5: A precisão, facilidade no entendimento e uso e o custo são os principais fatores que influenciam a escolha do modelo de previsão nas indústrias alimentícias pesquisadas.

A partir dos trabalhos de Winklhofer et al. (1996) e Fildes \& Hastings (1994), foram levantadas as principais dificuldades encontradas no uso de modelos de previsão de demanda (dificuldade no entendimento, dificuldades no uso, alto custo, alto tempo despendido, dificuldade na obtenção de dados, disponibilidade de softwares de precisão), chegando à Questão 3.

Questão 3: Quais as principais dificuldades encontradas no uso de modelos de previsão de demanda?

Hipótese 6: A dificuldade no entendimento e uso, além do alto custo, são os principais fatores que influenciam a escolha do modelo pelas indústrias alimentícias pesquisadas.

Além das variáveis encontradas na literatura que embasam a Questão 2, para a Questão 3 foi acrescentada a variável Tipo de produto, que pode sinalizar quais setores são mais deficientes e demandam novas pesquisas.

\section{Metodologia de pesquisa}

\subsection{Composição da amostra}

A partir da relação de indústrias associadas à ABIA em 2008, foram selecionadas todas as 450 empresas responsáveis pela produção exclusiva de alimentos. Os contatos foram feitos por telefone e e-mail, e dentre as empresas contatadas, 65 responderam o questionário por completo (índice de resposta de 14,44\%).

As empresas variam em tamanho, origem e localização, representando os diferentes setores da produção de alimentos, de acordo com divisão da ABIA (2008):

- derivados de carnes

- beneficiamento de café, chá e cereais

- óleos e gordura

- laticínios
- derivados de trigo

- açúcares

- derivados de frutas e vegetais

- chocolate, cacau e balas

- desidratados e supergelados

- conservas de pescados

\subsection{Coleta de dados}

A base para esta pesquisa foi obtida a partir de questionário (Anexo A), respondido por e-mail, por profissional responsável pelo planejamento de demanda.

\subsubsection{Estrutura do questionário}

O questionário está dividido em duas partes. A primeira parte tem como objetivo coletar dados de forma a possibilitar a caracterização das empresas, enquanto a segunda busca a caracterização do processo de planejamento de demanda.

Os dados coletados na primeira parte dizem respeito à localização da empresa, tamanho, tipo de produto e origem (nacional ou multinacional).

Já as principais características a serem identificadas no processo de previsão pelas empresas são: setor responsável, modelo utilizado (se sim), acurácia do modelo, horizonte de tempo, intervalo de previsão, fatores que influenciam a escolha do modelo e dificuldades no uso destes modelos.

A acurácia do modelo de previsão corresponde à porcentagem de acertos do modelo de previsão em relação à demanda real. De acordo com Armstrong (2001), a acurácia é um critério relevante para identificar o estágio de maturidade do processo de previsão. O horizonte de tempo é definido por Pellegrini (2000) como o número de períodos futuros no qual o processo de previsão será avaliado. De acordo com o mesmo autor, o intervalo de previsão corresponde à frequência ou período de tempo no qual novas previsões são calculadas (semanalmente, mensalmente, quinzenalmente). Nesse sentido, pode-se analisar se as metodologias que vêm sendo utilizadas para a implantação dos sistemas de previsão respondem aos objetivos esperados.

\subsection{Análise discriminante}

Para responder às questões de pesquisa e hipóteses especificadas na seção 2, a partir dos questionários respondidos, foi utilizada a análise discriminante. Para o tratamento estatístico, foi utilizado o software SPSS (Statistic Package for Social Study).

Segundo Fávero et al. (2009), a análise discriminante é uma técnica de dependência multivariada que pode 
ser usada quando a variável dependente é qualitativa (não métrica), enquanto as variáveis independentes são quantitativas (métricas). Caracteriza-se, portanto, como uma técnica de previsão e classificação.

Maroco (2011) apresenta os principais objetivos da técnica:

a) identificar as variáveis que melhor discriminam dois ou mais grupos estruturalmente diferentes e mutuamente exclusivos;

b) utilizar essas variáveis para criar uma função discriminante que represente de forma parcimoniosa as diferenças entre os grupos;

c) utilizar essa função discriminante para classificar, a priori, novos elementos nos grupos.

A análise discriminante tem como objetivo identificar as variáveis que possibilitem a diferenciação e o delineamento dos grupos, uma vez que se trabalha com amostragem heterogênea com um grande número de variáveis.

\subsubsection{Modelagem da análise discriminante}

A modelagem da análise discriminante descrita nesta seção foi extraída de Fávero et al. (2009).

Antes de iniciarmos a modelagem da análise discriminante propriamente dita, é pertinente esclarecermos os pressupostos inerentes a esta técnica. Pode-se dizer que há dois pressupostos principais referentes à existência de normalidade multivariada das variáveis explicativas e à presença de homogeneidade das matrizes de variância e covariância para os grupos.

Em relação à primeira suposição, a combinação linear das variáveis explicativas apresenta uma distribuição normal e, caso ocorra uma violação deste pressuposto, a $\mathrm{AD}$ poderá causar distorções nas avaliações do pesquisador, principalmente se a amostra que compõe cada grupo for pequena. No entanto, se esta violação somente ocorrer pela existência de assimetria da distribuição, a decisão sobre a aplicação da técnica não sofrerá alteração.

$\mathrm{O}$ segundo pressuposto refere-se à existência de homogeneidade das matrizes de variância e covariância. Este pressuposto é verificado por meio da estatística Box's M.

Além desses pressupostos, é pertinente ressaltar que a inexistência de outliers, a presença de linearidade das relações e a ausência de problemas relacionados à multicolinearidade das variáveis explicativas são também consideradas pressupostos da análise discriminante.

A análise discriminante permite o conhecimento das variáveis que mais se destacam na discriminação dos grupos. Para tanto, diversos outputs são gerados a partir de testes e estatísticas, como o lambda de Wilks, a correlação canônica, o Qui-quadrado e o autovalor (eigenvalue).
O lambda de Wilks, dado pela Equação 1, é uma medida que varia de 0 a 1 e propicia a avaliação da existência de diferenças de médias entre os grupos para cada variável; valores elevados desta estatística indicam ausência de diferenças entre os grupos.

$$
\Lambda=\frac{S Q_{d g}}{S Q T}
$$

em que $\mathrm{SQ}_{\mathrm{dg}}$ representa a soma dos erros (dentro dos grupos), e SQT a soma dos quadrados total.

Com a seleção das variáveis discriminantes (explicativas) para a formação dos grupos, passamos à identificação das funções discriminantes. A AD assemelha-se à análise de regressão em termos de objetivos e características e, dessa forma, a sua função geral pode ser representada por meio da equação linear (Equação 2):

$$
Z_{n}=\alpha+\beta_{1} X+\beta_{2} X_{2}+\ldots+\beta_{n} X_{n}
$$

em que:

Z: variável dependente;

$\alpha:$ intercepto;

$\mathrm{X}_{\mathrm{i}}$ : variáveis explicativas;

$\beta_{\mathrm{i}}$ : coeficientes discriminantes para cada variável explicativa.

É importante ressaltar que esta função discriminante é diferente da função discriminante linear de Fisher, uma vez que, enquanto a primeira é utilizada como um meio de facilitar a interpretação dos parâmetros das variáveis explicativas, a função discriminante linear de Fisher é utilizada para classificar as observações nos grupos.

Na função discriminante linear de Fisher, os valores das variáveis explicativas de uma dada observação são inseridos nas funções de classificação e, consequentemente, um escore de classificação é calculado para cada grupo, para aquela determinada observação.

Dadas $p$ variáveis e $g$ grupos, é possível estabelecer $m=\operatorname{mín}(g-1 ; p)$ funções discriminantes que são combinações lineares das $p$ variáveis, de modo que a função linear de Fisher é dada pela Equação 3:

$$
Z_{n}=W_{1} X_{1}+W_{2} X_{2}+\ldots+W_{n} X_{n}
$$

em que $\mathrm{W}_{\mathrm{i}}$ representa o vetor de pesos das variáveis para as funções discriminantes, e são estimados de modo que a variabilidade dos escores da função discriminante seja máxima entre os grupos e mínima dentro dos grupos (Maroco, 2011). Assim, podemos expressar o i-ésimo eigenvalue (i-ésima função discriminante) da seguinte forma (Equação 4):

$$
\text { eigenvalue }_{i}=\frac{S Q_{e g}}{S Q_{d g}}
$$

em que:

$\mathrm{SQ}_{\mathrm{eg}}$ : soma dos quadrados entre os grupos;

$\mathrm{SQ}_{\mathrm{dg}}$ : soma dos quadrados dentro dos grupos. 
Assim, eigenvalues altos resultam em boas funções discriminantes.

Expressa de acordo com a Equação 3, a função discriminante é conhecida por função discriminante linear de Fisher e, após a dedução da primeira função discriminante, os pesos $\mathrm{W}_{\mathrm{i}}$ das funções seguintes são obtidos sob a restrição adicional de que os escores das funções discriminantes não estejam correlacionados.

Por meio destes cálculos, é possível chegarmos à expressão da primeira função discriminante $\left(\mathrm{Z}_{1}\right)$; as outras funções dos outros grupos são encontradas pelo mesmo método. Porém, é preciso que tenhamos atenção em relação a problemas de correlação, ou seja, os escores das outras funções $\left(Z_{2}, Z_{3}, \ldots, Z_{n}\right)$ não devem ser correlacionados. Assim, o eigenvalue da segunda função discriminante linear de Fisher pode ser escrito a partir da Equação 5:

$$
\text { eigenvalue }=\frac{S Q_{e g}\left(Z_{2}\right)}{S Q_{d g}\left(Z_{2}\right)}
$$

em que:

$\mathrm{SQ}_{\mathrm{eg}}\left(\mathrm{Z}_{2}\right)$ : soma dos quadrados entre os grupos na $2^{\mathrm{a}}$ função discriminante;

$\mathrm{SQ}_{\mathrm{dg}}\left(\mathrm{Z}_{2}\right)$ : soma dos quadrados dentro dos grupos na $2^{\mathrm{a}}$ função discriminante.

Por meio das funções discriminantes lineares de Fisher, é possível estudarmos a influência que uma determinada variável dependente categórica sofre de um vetor de variáveis explicativas. Ademais, é possível estabelecer uma relação entre os eigenvalues e o lambda de Wilks, como mostra a Equação 6:

$$
\Lambda=\prod\left[\frac{1}{\left(1+\text { eigenvalue }_{i}\right)}\right]
$$

Para a existência de $g$ grupos, há (g-1) funções discriminantes. Assim, se tivéssemos, por exemplo, 3 grupos, seriam geradas 2 funções discriminantes, sendo a primeira responsável pela separação de um grupo dos demais e a segunda pela separação dos dois grupos restantes.

Outra estatística resultante refere-se à correlação canônica, que corresponde à razão entre a variação entre os grupos e a variação total, e mede o grau de associação entre os escores discriminantes e os grupos. A correlação canônica é dada pela Equação 7:

$$
\text { CANCOR }=\sqrt{\frac{S Q_{e g}}{S Q T}}
$$

que resulta na Equação 8:

$$
\Lambda+\text { CANCOR }^{2}=1
$$

Apresentamos agora as hipóteses nula e alternativa que se referem às médias populacionais dos grupos em análise para as variáveis explicativas. Para tanto, utilizamos a estatística da distribuição $F$ para testar as seguintes hipóteses:

$$
\begin{aligned}
& H_{0}=\mu_{1}=\mu_{2}=\ldots=\mu_{n} \\
& H_{0}=\mu_{1} \neq \mu_{2} \neq \ldots \neq \mu_{n}
\end{aligned}
$$

em que $\mu_{1}, \mu_{2}, \ldots, \mu_{\mathrm{n}}$ são as médias populacionais dos grupos $1,2, \ldots, \mathrm{n}$, respectivamente. A hipótese nula será rejeitada se pelo menos as médias de dois grupos for significativamente diferente (Maroco, 2011). Dessa forma, a hipótese alternativa, quando não rejeitada, indica que as variáveis explicativas apresentam médias diferentes entre os grupos.

Após a função discriminante ser definida, será calculado o escore discriminante da variável dependente $(Z)$ para cada observação, ou seja, os escores serão calculados de maneira a propiciar a definição do escore crítico que determinará a forma por meio da qual iremos classificar uma observação em um determinado grupo. Para grupos de mesma dimensão amostral (tamanho), o cálculo do escore de corte (cutoff value) é dado pela Equação 9:

$$
f=\frac{\bar{d}_{1}+\bar{d}_{2}}{2}
$$

em que $\bar{d}_{1}$ e $\bar{d}_{2}$ representam as médias das funções discriminantes (centroides) nos grupos 1 e 2, respectivamente. Já para grupos com tamanhos diferentes, temos (Equação 10):

$$
f=\frac{n_{1} \bar{d}_{1}+n_{2} \bar{d}_{2}}{n_{1}+n_{2}}
$$

em que $n_{1}$ e $n_{2}$ são os tamanhos dos grupos 1 e 2, respectivamente. Normalmente, o valor de corte selecionado é aquele que minimiza o numero de classificações incorretas. De acordo com Maroco (2011), um determinado caso é classificado no grupo 1 se o seu escore na função discriminante for maior que $f$. No caso de mais de 2 grupos, pode-se definir a zona de fronteira para cada par de grupos, conhecida por mapa territorial. Como os centroides representam as médias das funções discriminantes em cada grupo, temos, num determinado mapa territorial, que:

$$
\mathrm{k} \text { grupos }=\mathrm{k} \text { centroides }
$$

O método do cálculo do escore crítico é um dos procedimentos existentes para a classificação de futuras observações. Os outros métodos também podem ser mencionados, como o da Teoria da Decisão Estatística, o da Função de Classificação e o $\mathrm{D}^{2}$ de Mahalanobis.

Maroco (2011) descreve que a distância de cada escore ao centroide de um determinado grupo pode ser calculada a partir da Equação 11:

$$
D_{j}^{2}=\left(d-\bar{d}_{j}\right) S_{j}^{-1}\left(d-\bar{d}_{j}\right)^{\prime}
$$


em que $S_{j}^{-1}$ representa a matriz de variância e covariância para as funções discriminantes no grupo $g$ e $\bar{d}_{j}$ o centroide deste grupo. Se uma determinada observação pertencer ao grupo $(j=1, \ldots, g)$, então $D_{g}^{2}$ possui distribuição Qui-quadrado com m (número de funções discriminantes) graus de liberdade.

\section{Análise dos resultados}

Os resultados obtidos foram gerados pelos questionários respondidos pelas 65 empresas que concordaram em participar do trabalho, o que representa um índice de resposta de 14,4\%.

Os métodos estatísticos propostos foram aplicados para análise dos dados dos questionários levantados, de forma a responder às questões de pesquisa e testar as hipóteses descritas na seção 2, buscando atender cada objetivo específico.

\subsection{Caracterização das empresas}

Das empresas participantes, $62,5 \%$ era de pequeno porte, $20,3 \%$ de médio porte e $17,2 \%$ de grande porte. A maioria dessas empresas $(89,2 \%)$ é de capital nacional, sendo as multinacionais representadas por $10,8 \%$.

Com relação à localização, a maior participação foi de representantes da Região Sudeste do país, correspondendo a 73,85\%, seguida da Região Sul com 18,46\%, Região Nordeste com 6,15\%, Centro-Oeste com 1,54\%, não havendo representantes da Região Norte.

A produção principal das empresas participantes é mostrada por ordem de representatividade:

- óleos e gordura $-25,35 \%$

- laticínios - 15,48\%

- conservas de pescados $-12,68 \%$

- derivados de carnes $-11,27 \%$

- outros $-11,27 \%$

- beneficiamento de café, chá e cereais $-8,45 \%$

- derivados de frutas e vegetais $-7,04 \%$

- açúcares - 2,82\%

- desidratados e supergelados $-2,82 \%$

- derivados de trigo - $1,41 \%$

- chocolate, cacau e balas - $1,41 \%$

Conclui-se que a maior participação nessa pesquisa foi de empresas de pequeno porte, de capital nacional, localizadas na Região Sudeste e produtores em sua maioria de óleos e gordura.

\subsection{Estatística descritiva para analisar os modelos de previsão que vêm sendo utilizados pelas empresas}

Através de estatística descritiva, buscou-se responder à primeira questão de pesquisa e rejeitar ou não as hipóteses 1, 2, 3 e 4.

Os resultados das estatísticas descritivas comparando os modelos de previsão de demanda utilizados pelas empresas estão apresentados a seguir.

A distribuição de frequências está ilustrada na Tabela 1.

A Tabela 1 mostra que o modelo de Análise histórica é o mais utilizado (35 empresas), seguido pelo modelo de Análise de mercado (13 empresas) e o modelo de média móvel (13 empresas).

Desta forma, conclui-se que a hipótese 1 é verdadeira, ou seja, as indústrias pesquisadas utilizam diversos modelos de previsão de demanda dentre os listados no questionário.

A partir dos resultados apresentados, conclui-se também que a hipótese 2 é verdadeira, ou seja, a maioria das empresas pesquisadas fornecedoras de produtos alimentícios não utiliza modelos sofisticados de previsão, pois $93,8 \%$ das empresas pesquisadas usam Análise histórica, Média móvel ou Análise de mercado. Pode-se concluir também que, diferentemente da pesquisa realizada por Jain \& Malehorn (2006), o modelo de Alisamento exponencial não aparece nesse trabalho como o mais utilizado para previsão de demanda, rejeitando-se a hipótese 3.

Os resultados também mostram que não existe um modelo único de previsão a ser utilizado por todas as indústrias pesquisadas, confirmando a hipótese 4 elaborada a partir dos trabalhos de Jain \& Malehorn (2006) e Zhou (1999).

\subsection{Análise discriminante para avaliar os principais fatores que influenciam a escolha do modelo}

A técnica de análise discriminante (AD) será utilizada para avaliar a influência de diversas variáveis (Horizonte de previsão, Facilidade no entendimento, Facilidade no uso, Custo, Tempo despendido, Precisão, Consistência dos dados, Disponibilidade de softwares estatísticos e Tipo de produto) na escolha dos modelos de previsão. As variáveis independentes são quantitativas ou métricas (nota de 0 a 10 em função de sua importância) e a variável dependente é categórica, qualitativa ou não métrica (grupos de métodos utilizados). Dessa forma, a técnica de análise discriminante pode ser aplicada para verificar a influência de cada variável independente na discriminação dos grupos. 
O Quadro 1 mostra o nome das variáveis pesquisadas, o nome adotado na rotina, a escala adotada e o tipo de variável.

O modelo considera como variável dependente o modelo de previsão e as variáveis preditoras ou independentes na análise são: Horizonte de previsão, Facilidade no entendimento, Facilidade no uso, Custo, Tempo despendido, Precisão, Consistência dos dados, Disponibilidade de softwares estatísticos e Tipo de produto. No entanto, quando todas as variáveis são consideradas no processo de análise, identificou-se que as variáveis Horizonte de previsão, Facilidade no entendimento, Facilidade no uso, Custo, Precisão, Consistência dos dados e Disponibilidade de softwares estatísticos não foram significativas para o modelo estatístico, o que prejudicaria os resultados da análise. A partir dessa verificação, outro modelo de análise discriminante foi construído apenas com as seguintes variáveis preditoras (independentes): Tempo despendido e Tipo de produto. Os resultados seguem abaixo, com base no roteiro proposto por Fávero et al. (2009).

Primeiramente, por meio do teste de lambda de Wilks que testa as diferenças entre as médias dos grupos para cada variável analisada, verificou-se que

Tabela 1. Distribuição de frequências.

\begin{tabular}{llcccc}
\hline & Frequência & Porcentagem & $\begin{array}{c}\text { Porcentagem } \\
\text { válida }\end{array}$ & $\begin{array}{c}\text { Porcentagem } \\
\text { cumulativa }\end{array}$ \\
\hline Valid & simulacao & 1 & 1,5 & 1,5 & 1,5 \\
& analise_mercado & 13 & 20,0 & 20,0 & 21,5 \\
& Delphi & 1 & 1,5 & 1,5 & 23,1 \\
Analise_historica & 35 & 53,8 & 53,8 & 76,9 \\
Media_movel & 13 & 20,0 & 20,0 & 96,9 \\
Alisamento_exponencial & 1 & 1,5 & 1,5 & 98,5 \\
Arima & 1 & 1,5 & 1,5 & 100,0 \\
& Total & 65 & 100,0 & 100,0 & \\
\hline
\end{tabular}

Tabela gerada pelo programa SPSS.

Quadro 1. Variáveis pesquisadas.

\begin{tabular}{|c|c|c|c|}
\hline Variáveis pesquisadas & Variável Nome & Escala adotada & Tipo de Escala \\
\hline \multirow{13}{*}{ Modelo } & \multirow{13}{*}{ Modelo } & $0=$ não utiliza & \multirow{13}{*}{ Nominal } \\
\hline & & $1=$ simulação & \\
\hline & & $2=$ análise de mercado & \\
\hline & & 3 = Delphi & \\
\hline & & $4=$ painel de especialista & \\
\hline & & $5=$ análise histórica & \\
\hline & & 6 = média móvel & \\
\hline & & 7 = alisamento exponencial & \\
\hline & & $8=$ arima & \\
\hline & & $9=$ decomposição & \\
\hline & & $10=$ regressão & \\
\hline & & 11 = econométrico & \\
\hline & & $12=$ redes neurais & \\
\hline Horizonte de previsão & Horizonte_previsao & Numérica/inteiro(0 a 10) & Métrica \\
\hline Facilidade no entendimento & Facilidade_entendimento & Numérica/inteiro(0 a 10) & Métrica \\
\hline Facilidade no uso & Facilidade_uso & Numérica/inteiro(0 a 10) & Métrica \\
\hline Custo & Custo & Numérica/inteiro(0 a 10) & Métrica \\
\hline Tempo despendido & Tempo_despendido & Numérica/inteiro(0 a 10) & Métrica \\
\hline Precisão & Precisao & Numérica/inteiro(0 a 10) & Métrica \\
\hline Consistência dos dados & Consistencia_dados & Numérica/inteiro(0 a 10) & Métrica \\
\hline Disponibilidade de softwares estatísticos & Disponibilidade_software & Numérica/inteiro(0 a 10) & Métrica \\
\hline Tipo de produto & Tipo_produto & Numérica/inteiro(0 a 10) & Métrica \\
\hline
\end{tabular}


há igualdade entre os grupos para ambas as variáveis, já que esses valores estão próximos de 1.

Por meio do teste F, que parte da hipótese nula de que a variável não é significativa para discriminação dos grupos, verificou-se, primeiramente, que a variável Tempo despendido não possui significância para o modelo estatístico. Por outro lado, a hipótese foi rejeitada para a variável Tipo de produto, indicando seu poder discriminatório para análise.

Analisando as matrizes de covariância e correlação entre as variáveis, verificou-se uma ausência de correlação entre estas.

A identificação da presença de homogeneidade de covariância, uma das hipóteses da análise discriminante, foi verificada a partir do teste de Box's M. A hipótese nula do teste de Box's M afirma que não há diferenças significativas entre os grupos ou que as matrizes de covariância dos grupos são homogêneas. Concluímos que há igualdade nas dispersões dos grupos, já que a hipótese nula não foi rejeitada.

Os eigenvalues para as funções discriminantes compõem o percentual de explicação da variância entre os grupos. Se os eigenvalues estão próximos de 1 , representam pouca variação entre os grupos, explicada pela função descriminante; eigenvalues distantes de 1 representam grandes variações entre os grupos, explicadas pela função discriminante (Fávero et al., 2009). Neste caso, a função 1 explica $72,4 \%$ da variância entre os grupos, enquanto a função 2 explica apenas $27,6 \%$.

Pelo lambda de Wilks e pelo teste qui-quadrado, confirmou-se que pelo menos a primeira função discriminante é significativa, o que não acontece com a segunda função.

Já os coeficientes padronizados das funções discriminantes avaliam a importância de cada variável para a função discriminante, também podendo ser chamados de coeficientes de pesos discriminantes (Maroco, 2011). A Tabela 2 apresenta os coeficientes padronizados das funções discriminantes.

Analisando os pesos discriminantes, segundo Maroco (2011), verifica-se que a variável Tipo de produto possui maior coeficiente positivo para a primeira função discriminante, enquanto a variável Tempo despendido possui maior coeficiente positivo para a segunda função.

A matriz de estrutura descrita na Tabela 3 informa as correlações entre as variáveis independentes e as funções discriminantes canônicas padronizadas.

Pode-se notar, a partir da Tabela 3, a existência de asteriscos $\left(^{*}\right)$ em algumas variáveis. Esses asteriscos indicam as variáveis mais importantes para a determinação das funções discriminantes, ou as maiores correlações absolutas entre cada variável e suas funções discriminantes (Fávero et al., 2009). Verifica-se que a variável Tipo de produto possui maior correlação positiva com a primeira função discriminante, enquanto Tempo despendido possui alta correlação positiva com a segunda função.

A Tabela 4 mostra os centroides dos grupos.

Na Tabela 5, têm-se os coeficientes de classificação que permitem criar modelos para classificar novas observações ou empresas para o estudo.

Para esse estudo, pode-se criar um modelo matemático para cada modelo de previsão, no entanto, alguns dos modelos analisados possuíam poucas informações, sendo esses resultados insuficientes para criar suas funções (amostras com 1 única observação).

Na Figura 1 tem-se a representação gráfica dos centroides de cada grupo nas funções discriminantes.

Essa representação gráfica inclui todos os possíveis modelos, alguns prejudicados pelo fato de terem poucas observações (simulação, alisamento exponencial, Delphi e ARIMA), podendo gerar classificações incorretas.

A porcentagem de classificação correta do modelo estudado foi de $47,7 \%$. Esse valor pode ser explicado pelo pequeno número de variáveis independentes considerado no modelo, já que as demais variáveis não se mostraram significativas. Por outro lado, a variável Tipo de produto mostrou-se altamente significativa na escolha do modelo.

Tabela 2. Coeficientes padronizados das funções discriminantes.

\begin{tabular}{lcc}
\hline & \multicolumn{2}{c}{ Função } \\
\cline { 2 - 3 } & $\mathbf{1}$ & $\mathbf{2}$ \\
\hline Tempo_despendido &, 544 &, 848 \\
Tipo_produto &, 905 &,- 441 \\
\hline
\end{tabular}

Tabela 3. Matriz de estrutura.

\begin{tabular}{lll}
\hline & \multicolumn{2}{c}{ Função } \\
\cline { 2 - 3 } & \multicolumn{1}{c}{$\mathbf{2}$} & $\mathbf{2}$ \\
\hline Tipo_produto &, $842\left(^{*}\right)$ &,- 540 \\
Tempo_despendido &, 438 &, $89\left(^{*}\right)$ \\
\hline
\end{tabular}

*Maior correlação absoluta entre cada variável e qualquer função discriminante

Tabela 4. Centroides dos grupos.

\begin{tabular}{lcc}
\hline \multirow{2}{*}{ Modelo } & \multicolumn{2}{c}{ Função } \\
\cline { 2 - 3 } & $\mathbf{1}$ & $\mathbf{2}$ \\
\hline Não utiliza &,- 972 &,- 793 \\
Simulação &,- 115 &, 335 \\
Análise de mercado &,- 191 &, 363 \\
Delphi &,- 593 & 1,051 \\
Análise histórica &, 932 &,- 164 \\
Média móvel &,- 795 &,- 203 \\
Alisamento exponencial &,- 069 &,- 846 \\
Arima &, 467 &, 825 \\
Regressão &, 796 &, 085 \\
\hline
\end{tabular}


Tabela 5. Coeficientes de classificação das funções discriminantes.

\begin{tabular}{lrrrrrrrrr}
\hline & \multicolumn{10}{c}{ Modelo } \\
\cline { 2 - 10 } & $\begin{array}{c}\text { Não } \\
\text { utiliza }\end{array}$ & Simulação & $\begin{array}{c}\text { Análise de } \\
\text { mercado }\end{array}$ & Delphi & $\begin{array}{c}\text { Analise } \\
\text { histórica }\end{array}$ & $\begin{array}{c}\text { Média } \\
\text { móvel }\end{array}$ & $\begin{array}{c}\text { Alisamento } \\
\text { exponencial }\end{array}$ & Arima & Regressão \\
\hline Tempo_despendido & 1,482 & 2,223 & 2,214 & 2,404 & 2,299 & 1,793 & 1,714 & 2,604 & 2,371 \\
Tipo_produto & 1,368 & 1,557 & 1,502 & 1,051 & 2,347 & 1,301 & 1,937 & 1,768 & 2,189 \\
(Constant) & $-8,203$ & $-13,674$ & $-11,562$ & $-14,486$ & $-15,190$ & $-8,681$ & $-11,484$ & $-18,545$ & $-18,036$ \\
\hline
\end{tabular}

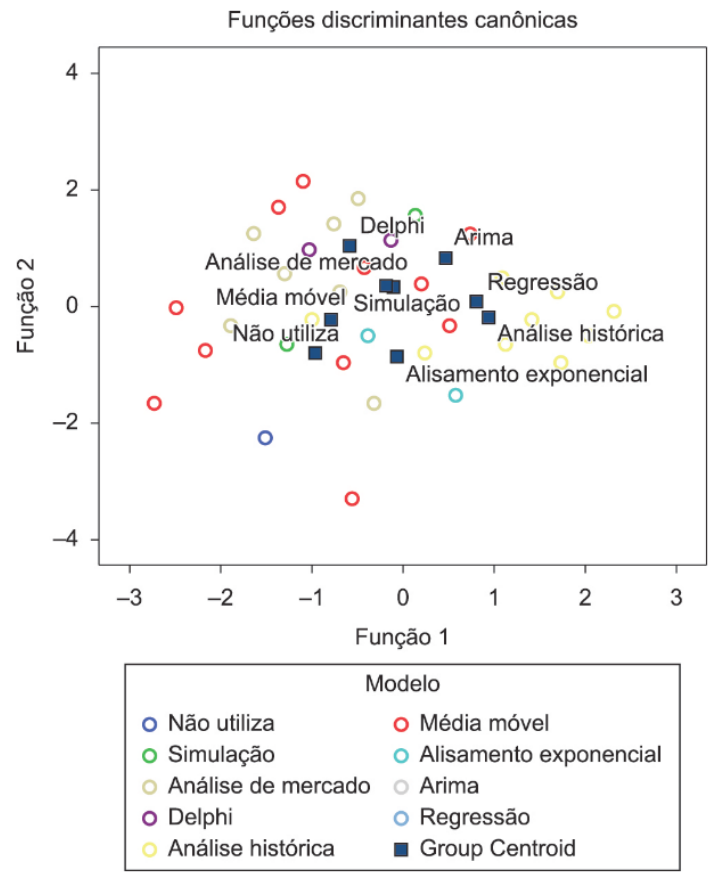

Figura 1. Representação gráfica dos centroides nas funções discriminantes.

Pode-se concluir que a variável Tipo de produto é a que mais influencia na escolha dos modelos de previsão de demanda, seguida pela variável Tempo despendido. Nota-se também que os modelos de Análise histórica e Regressão são aqueles com maiores coeficientes discriminantes positivos para a variável Tipo de produto, enquanto as empresas que não utilizam nenhum modelo de previsão e as que utilizam Média móvel são aquelas com maiores coeficientes discriminantes negativos para a mesma variável. Já os modelos Delphi e ARIMA são aqueles com maiores coeficientes discriminantes positivos para a variável Tempo despendido, enquanto as empresas que não utilizam modelos de previsão e as que utilizam o modelo de Alisamento exponencial são as que apresentam menores coeficientes discriminantes positivos. Conclui-se, portanto, que as empresas que utilizam o modelo de Análise histórica e Regressão consideram a variável Tipo de produto como o fator principal na escolha do modelo, enquanto as empresas que utilizam o modelo de Média móvel não consideram a variável Tipo de produto como o fator principal na escolha do modelo. Conclui-se também que as empresas que utilizam os modelos Delphi e ARIMA consideram a variável Tempo despendido como fator principal na escolha do modelo, enquanto as empresas que utilizam o modelo de Alisamento exponencial não consideram a variável Tempo despendido como o fator principal na escolha do modelo.

Dessa forma, a hipótese 5, que afirma que as variáveis Precisão, Facilidade no entendimento e uso e o Custo são os principais fatores que influenciam a escolha do modelo nas indústrias alimentícias brasileiras, é rejeitada, já que elas não se mostraram significantes no modelo estatístico de análise discriminante, cabendo a maior importância para a variável Tipo de produto.

\subsection{Análise discriminante para avaliar as principais dificuldades encontradas no uso de modelos de previsão de demanda}

Nesta seção, será utilizada a técnica de análise discriminante (AD) para avaliar as principais dificuldades encontradas (Dificuldade no entendimento, Dificuldade no uso, Alto custo, Alto tempo despendido, Dificuldade na obtenção dos dados, Disponibilidade de softwares estatísticos, Tipo de produto da empresa, Falta de mão de obra específica e Falta de interesse) no uso de modelos de previsão. As variáveis independentes são quantitativas ou métricas (nota de 0 a 10 em função de sua importância), e a variável dependente é categórica, qualitativa ou não métrica (grupos de modelos utilizados), o que justifica a aplicação da técnica de análise discriminante para determinar a influência de cada variável independente na discriminação dos grupos.

O Quadro 2 mostra as variáveis pesquisadas para essa questão, o nome adotado no software SPSS, a escala adotada e seu tipo.

O modelo considera como variável dependente o Modelo de previsão, e as variáveis preditoras ou independentes na análise são: Dificuldade no entendimento, Dificuldade no uso, Alto custo, Alto tempo despendido, Dificuldade na obtenção dos dados, Disponibilidade de softwares estatísticos, Tipo de produto da empresa, Falta de mão de obra 
específica, Falta de interesse. No entanto, quando todas as variáveis são consideradas no processo de análise, identificou-se que as variáveis Dificuldade no uso, Alto custo, Alto tempo despendido, Dificuldade na obtenção dos dados, Tipo de produto da empresa, Falta de mão de obra específica e Falta de interesse não foram significativas para o modelo estatístico, o que prejudicaria os resultados da análise. A partir dessa verificação, outro modelo de análise discriminante foi construído apenas com as seguintes variáveis preditoras (independentes): Disponibilidade de softwares estatísticos e Dificuldade no entendimento, sendo a variável resposta ou dependente o modelo de previsão. Os resultados seguem abaixo.

A partir do teste de igualdade de médias (ANOVA), verificou-se que o valor de lambda de Wilks é próximo de 1 , indicando a ausência de diferenças entre os grupos. Já a hipótese nula do teste $\mathrm{F}$ de que a média dos grupos são iguais foi rejeitada para a variável Disponibilidade de software. O mesmo ocorre para a variável Dificuldade no atendimento. Verifica-se que o maior poder discriminatório pertence à variável Disponibilidade de software, sendo seu valor de lambda de Wilks menor comparado com a variável Dificuldade no atendimento. Conclui-se, portanto, que para ambas as variáveis preditoras existe pelo menos um grupo em que as médias são diferentes.

Por meio da matriz de correlação, verificam-se baixos valores de correlação entre as variáveis analisadas, indicando ausência de correlação entre elas.

A estatística Box's M foi aplicada para identificar a homogeneidade de covariância, concluindo que não há significância das diferenças observadas (Fávero et al., 2009), atendendo a um dos pressupostos da análise discriminante.

Os eigenvalues para as funções discriminantes $1 \mathrm{e}$ 2 foram 0,650 e 0,145 , respectivamente. Verificou-se que a função 1 explica $81,8 \%$ da variância entre os grupos, enquanto a função 2 explica apenas $18,2 \%$.

Pelo lambda de Wilks e pelo teste qui-quadrado, conclui-se que a primeira função tem um poder discriminante maior que a segunda função.

A Tabela 6 apresenta os coeficientes padronizados das funções discriminantes.

Verificando os pesos discriminantes, observa-se que a variável Disponibilidade de software possui um alto coeficiente positivo para a primeira

Quadro 2. Variáveis pesquisadas.

\begin{tabular}{|c|c|c|c|}
\hline Variáveis pesquisadas & $\begin{array}{c}\text { Nome da variável utilizada no } \\
\text { software }\end{array}$ & Escala adotada & Tipo de Escala \\
\hline \multirow{13}{*}{2 - Modelo } & \multirow{13}{*}{ Modelo } & $0=$ não utiliza & \multirow{13}{*}{ Nominal } \\
\hline & & $1=$ simulação & \\
\hline & & $2=$ análise de mercado & \\
\hline & & $3=$ Delphi & \\
\hline & & $4=$ painel de especialista & \\
\hline & & $5=$ análise histórica & \\
\hline & & $6=$ média móvel & \\
\hline & & $7=$ alisamento exponencial & \\
\hline & & $8=$ arima & \\
\hline & & $9=$ decomposição & \\
\hline & & $10=$ regressão & \\
\hline & & $11=$ econométrico & \\
\hline & & $12=$ redes neurais & \\
\hline Dificuldade no entendimento & Dificuldade_entendimento & Numérica/inteiro(0 a 10) & Métrica \\
\hline Dificuldade no uso & Dificuldade_uso & Numérica/inteiro(0 a 10) & Métrica \\
\hline Alto custo & Alto_custo & Numérica/inteiro(0 a 10) & Métrica \\
\hline Alto tempo despendido & Alto_tempo_despendido & Numérica/inteiro(0 a 10) & Métrica \\
\hline $\begin{array}{l}\text { Dificuldade na obtenção dos } \\
\text { dados }\end{array}$ & Dificuldade_dados & Numérica/inteiro(0 a 10) & Métrica \\
\hline $\begin{array}{l}\text { Disponibilidade de softwares } \\
\text { estatísticos }\end{array}$ & Disponibilidade_soft & Numérica/inteiro(0 a 10) & Métrica \\
\hline Tipo de produto da empresa & Tipo_produto & Numérica/inteiro(0 a 10) & Métrica \\
\hline $\begin{array}{l}\text { Falta de mão de obra } \\
\text { específica }\end{array}$ & Falta_mao_obra & Numérica/inteiro(0 a 10) & Métrica \\
\hline Falta de interesse & Falta_interesse & Numérica/inteiro(0 a 10) & Métrica \\
\hline
\end{tabular}


função discriminante. Já a variável Dificuldade no entendimento possui um alto coeficiente positivo para a segunda função discriminante.

A matriz de estrutura apresentada na Tabela 7 apresenta as correlações entre as variáveis independentes e as funções discriminantes canônicas padronizadas.

Pela Tabela 8, pode-se verificar que a variável Disponibilidade de software possui maior correlação positiva com a primeira função discriminante. Já a variável Dificuldade no entendimento possui alta correlação positiva com a segunda função discriminante.

A Tabela 8 mostra os centroides dos grupos.

Na Tabela 9 têm-se os coeficientes de classificação que permitem criar modelos para classificar novos casos.

Na Figura 2 tem-se a representação gráfica dos centroides para cada grupo nas funções discriminantes.

Tabela 6. Coeficientes padronizados das funções discriminantes.

\begin{tabular}{lcc}
\hline & \multicolumn{2}{c}{ Função } \\
\cline { 2 - 3 } & $\mathbf{1}$ & $\mathbf{2}$ \\
\hline Disponibilidade_soft &, 810 &,- 586 \\
Dificuldade_entendimento &, 591 &, 806 \\
\hline
\end{tabular}

Tabela 7. Matriz de estrutura.

\begin{tabular}{lcc}
\hline & \multicolumn{2}{c}{ Função } \\
\cline { 2 - 3 } & $\mathbf{1}$ & $\mathbf{2}$ \\
\hline Disponibilidade_soft &, $806\left(^{*}\right)$ &,- 591 \\
Dificuldade_entendimento &, 586 &, $810\left(^{*}\right)$ \\
\hline * Maior correlação absoluta entre cada variável e qualquer \\
função discriminante.
\end{tabular}

Tabela 8. Centroides dos grupos.

\begin{tabular}{lcc}
\hline \multirow{2}{*}{ Modelo } & \multicolumn{2}{c}{ Função } \\
\cline { 2 - 3 } & $\mathbf{1}$ & $\mathbf{2}$ \\
\hline Não utiliza & 1,262 &, 718 \\
Simulação &, 658 &,- 376 \\
Análise de mercado &,- 232 &,- 178 \\
Delphi & $-1,149$ &,- 532 \\
Análise histórica &, 082 &,- 162 \\
Média móvel &, 669 &, 249 \\
Alisamento exponencial & $-2,063$ &, 529 \\
Arima & $-2,331$ & 1,521 \\
Regressão & $-1,153$ &,- 130 \\
\hline
\end{tabular}

A porcentagem de classificação correta de casos para o estudo em questão foi de $40 \%$. Esse valor pode ser explicado pelo pequeno número de variáveis independentes consideradas na análise, já que as demais variáveis não se mostraram significativas. Porém, verificou-se que as variáveis Disponibilidade de software e Dificuldade no entendimento influenciam significativamente o uso de modelos de previsão de demanda, sendo que a primeira variável possui maior poder discriminatório.

Pode-se concluir que a variável Disponibilidade de software é a principal dificuldade no uso de modelos de previsão de demanda, seguida pela variável Dificuldade no entendimento. Nota-se também que as empresas que não utilizam modelos de previsão, juntamente com as que utilizam modelos de Simulação e Média móvel são aqueles com maiores coeficientes discriminantes positivos para a variável Disponibilidade de software, enquanto as empresas que utilizam os modelos ARIMA e Alisamento exponencial são aquelas com maiores coeficientes discriminantes negativos para a mesma variável. Já o modelo ARIMA é aquele com maior coeficiente

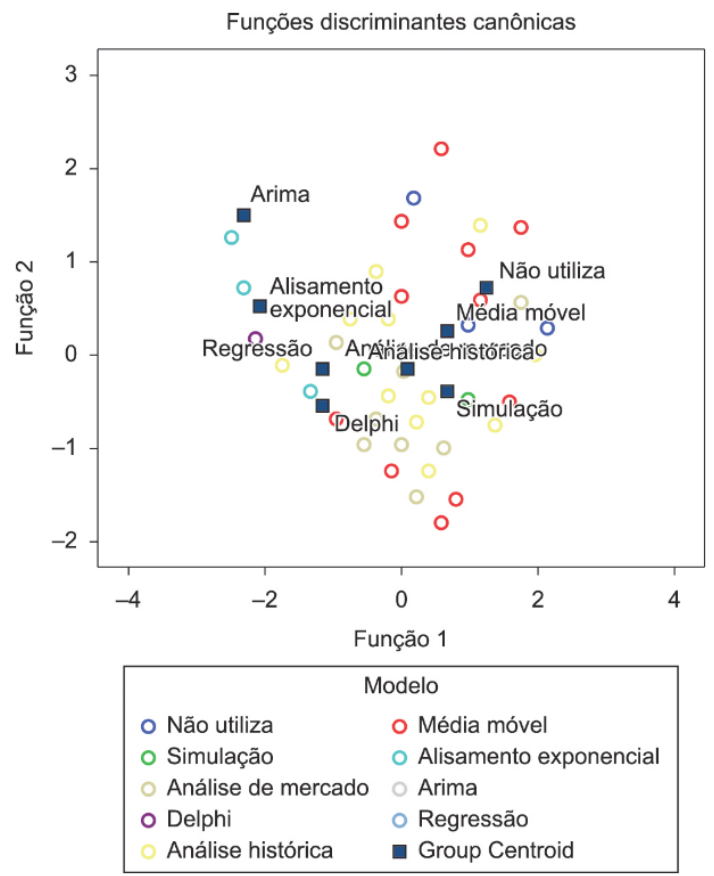

Figura 2. Representação gráfica dos centroides nas funções discriminantes.

Tabela 9. Coeficientes de classificação das funções discriminantes.

\begin{tabular}{lrcrrrrrrrr}
\hline & \multicolumn{10}{c}{ Modelo } \\
\cline { 2 - 10 } & $\begin{array}{c}\text { Não } \\
\text { utiliza }\end{array}$ & Simulação & $\begin{array}{c}\text { Análise de } \\
\text { mercado }\end{array}$ & Delphi & $\begin{array}{c}\text { Analise } \\
\text { histórica }\end{array}$ & $\begin{array}{c}\text { Media } \\
\text { móvel }\end{array}$ & $\begin{array}{c}\text { Alisamento } \\
\text { exponencial }\end{array}$ & Arima & Regressão \\
\hline Disponibilidade_soft & 1,864 & 1,937 & 1,534 & 1,276 & 1,652 & 1,765 &, 620 &, 235 & 1,161 \\
Dificuldade_entendimento &, 793 &, 392 &, 273 &, 006 &, 338 &, 557 &, 107 &, 315 &, 110 \\
(Constant) & $-13,216$ & $-11,867$ & $-6,590$ & $-6,991$ & $-7,632$ & $-9,843$ & $-3,956$ & $-4,764$ & $-7,132$ \\
\hline
\end{tabular}


discriminante positivo para a variável Dificuldade no entendimento. Conclui-se, portanto, que as empresas que utilizam o modelo de Simulação e Média móvel consideram a variável Disponibilidade de software como a principal dificuldade no uso de modelos de previsão, enquanto as empresas que utilizam o modelo ARIMA e Alisamento exponencial não consideram a variável Disponibilidade de software como a principal dificuldade. Conclui-se também que as empresas que utilizam o modelo ARIMA consideram a variável Dificuldade no entendimento como a principal dificuldade no uso do modelo.

Baseado nos resultados da análise discriminante, pode-se concluir que a hipótese 6 não pode ser totalmente confirmada, já que a principal variável que influencia a escolha de modelos de previsão pelas indústrias alimentícias brasileiras é a Disponibilidade de software que não havia sido mencionada. Por outro lado, a variável Dificuldade no atendimento, listada na hipótese 6 , também influencia diretamente a escolha dos modelos de previsão, porém, possui um menor poder discriminatório. Já a variável Alto custo não foi significativa para a escolha de modelos de previsão pelas indústrias alimentícias brasileiras.

\section{Conclusão}

Este trabalho apresentou uma análise das características, escolhas, utilizações e dificuldades das indústrias de alimentos pesquisadas diante da complexa tarefa que envolve o planejamento de demanda.

De acordo com os resultados apresentados, a amostra contemplou todos os setores produtivos de alimentos, sendo o de óleos e gordura o que teve maior representatividade, seguido do setor de laticínios. A região com maior número de indústrias participantes foi a Sudeste, seguida da Região Sul, e as demais não tiveram participações significativas. A maior participação nessa pesquisa foi de empresas de pequeno e médio porte, a maioria delas empresas nacionais.

Diferente da pesquisa americana realizada por Jain \& Malehorn (2006), que apontou o modelo de alisamento exponencial como o mais utilizado pelas indústrias do setor, as indústrias participantes optam, em sua maioria, por utilizar o modelo de análise histórica, seguido pelo modelo de análise de mercado e média móvel. Os modelos de simulação, Delphi, alisamento exponencial e ARIMA são os menos utilizados.

Pode-se concluir que a variável Tipo de produto é a que mais influencia na escolha dos modelos de previsão de demanda, seguida pela variável Tempo despendido. Conclui-se também que as empresas que utilizam o modelo de Análise histórica e Regressão consideram a variável Tipo de produto como o fator principal na escolha do modelo, enquanto as empresas que utilizam o modelo de Média móvel não consideram a variável Tipo de produto como o fator principal na escolha do modelo. Além disso, as empresas que utilizam os modelos Delphi e ARIMA consideram a variável Tempo despendido como fator principal na escolha do modelo, enquanto as empresas que utilizam o modelo de Alisamento exponencial não consideram a variável Tempo despendido como o fator principal na escolha do modelo.

As maiores dificuldades apontadas pelas empresas entrevistadas na utilização dos modelos de previsão de demanda foram: a Disponibilidade de software e a Dificuldade de entendimento dos modelos de previsão. Conclui-se também que as empresas que utilizam o modelo de Simulação e Média móvel consideram a variável Disponibilidade de software como a principal dificuldade no uso de modelos de previsão, enquanto as empresas que utilizam o modelo ARIMA e Alisamento exponencial não consideram a variável Disponibilidade de software como a principal dificuldade. Além disso, as empresas que utilizam o modelo ARIMA consideram a variável Dificuldade no entendimento como a principal dificuldade no uso do modelo.

Como limitações da pesquisa, a baixa frequência de empresas que utilizam modelos como simulação, Delphi, alisamento exponencial e Arima dificulta o processo de inferência. Como novos estudos, o mesmo trabalho pode ser aplicado para uma amostra de maior dimensão, agregando empresas que fazem parte de toda cadeia produtiva no setor de alimentos, o que permitirá explorar diferentes nuances do estágio atual do planejamento de demanda desse segmento produtivo brasileiro. O trabalho também pode ser aplicado para outros setores da indústria. Novas variáveis como o desconhecimento da técnica podem ser incluídas como possíveis motivos para a não utilização de modelos de previsão.

\section{Referências}

Armstrong, J. S. (2001). Evaluating forecasting methods. In J. S. Armstrong (Ed.), Principles of forecasting: handbook for researchers and practitioners (pp. 365-385). Norwell: Kluwer Academic Publishers.

Armstrong, J. S., \& Fildes, R. (2006). Making progress in forecasting. International Journal of Forecasting, 22(3), 433-441. http://dx.doi.org/10.1016/j.ijforecast.2006.04.007.

Associação Brasileira das Indústrias da Alimentação ABIA (2008). Anuário ABIA. São Paulo: Segmento Mc Editores Ltda.

Birchal, S. O. (2004). Empresa e indústria alimentícia no Brasil. Recuperado em 01 de março de 2009, de http://www.ceaee.ibmecmg.br/wp/wp17.pdf

Byrne, T. M. M., Moon, M. A., \& Mentzer, J. T. (2011). 
Motivating the industrial sales force in the sales forecasting process. Industrial Marketing Management, 40(1), 128-138. http://dx.doi.org/10.1016/j.indmarman.2010.06.003.

Conceição, J. C. P. R. (2007). Radiografia da indústria de alimentos no Brasil: identificação dos principais fatores referentes à exportação, inovação e ao food safety. Recuperado em 01 de março de 2009, de http://www.ipea.gov.br

Fávero, L. P., Belfiore, P., Silva, F. L., \& Chan, B. L. (2009). Análise de dados: modelagem multivariada para tomada de decisões. Rio de Janeiro: Campus Elsevier.

Fildes, R., \& Hastings, R. (1994). The organization and improvement of market forecasting. The Journal of the Operational Research Society, 45(1), 1-16. http:// dx.doi.org/10.1057/jors.1994.1.

Instituto Brasileiro de Geografia e Estatística - IBGE (2007). Censo 2007. Recuperado em 29 de junho 2008, de http://ibge.com.br

Jain, C. L., \& Malehorn, J. (2006). Benchmarking forecasting practices: a guide to improving forecasting performance. New York: Graceway Publishing Company.

Küsters, U., McCullough, B. C., \& Bell, M. (2006). Forecasting software: Past, present and future. International Journal of Forecasting, 22(3), 599-615. http://dx.doi.org/10.1016/j.ijforecast.2006.03.004.
Maroco, J. (2011). Análise estatística com a utilização do SPSS (5 ed.). Lisboa: Sílabo.

Naylor, T. H. (1981). Experience with corporate econometric models: a survey. Business Economics, 16(1), 79.

Pellegrini, F. R. (2000). Metodologia para implantação de sistemas de previsão de demanda (Dissertação de mestrado). Escola de Engenharia, Universidade Federal do Rio Grande do Sul, Porto Alegre.

Taylor, D. H., \& Fearne, A. (2009). Demand management in fresh food value chains: a framework for analysis and improvement. Supply Chain Management: An International Journal, 14(5), 379-392. http://dx.doi.org/10.1108/13598540910980297.

Wilson, J. H., \& Daubek, H. G. (1989). Marketing managers evaluate forecasting models. The Journal of Business Forecasting Methods \& Systems, 8(1), 19-22.

Winklhofer, H., Diamantopoulos, A., \& Witt, S. F. (1996). Forecasting practice: a review of the empirical literature and an agenda for future research. International Journal of Forecasting, 12(2), 193-221. http://dx.doi.org/10.1016/0169-2070(95)00647-8.

Zhou, W. (1999). Integration of different forecasting models. The Journal of Business Forecasting Methods \& Systems, 18(3), 26-31. 
Anexo A. Questionário de pesquisa.

\begin{tabular}{|c|c|c|}
\hline \multicolumn{3}{|c|}{$\begin{array}{l}\text { QUESTIONÁRIO DE PESQUISA: } \\
\text { A presente pesquisa acadêmica tem como objetivo aprofundar conhecimentos a respeito da previsão de } \\
\text { demanda nas indústrias de alimentos. }\end{array}$} \\
\hline \multirow{26}{*}{$\begin{array}{l}\text { CARACTERIZAÇÃO } \\
\text { EMPRESA }\end{array}$} & \multicolumn{2}{|c|}{ Nome da empresa: } \\
\hline & \multicolumn{2}{|l|}{ Nome do entrevistado: } \\
\hline & \multicolumn{2}{|c|}{ Tempo de experiência na empresa: } \\
\hline & \multicolumn{2}{|c|}{ Cargo do entrevistado: } \\
\hline & \multicolumn{2}{|c|}{ Tempo de experiência no cargo atual: } \\
\hline & \multicolumn{2}{|c|}{ Telefone: } \\
\hline & \multicolumn{2}{|c|}{ Email: } \\
\hline & \multicolumn{2}{|c|}{ Faturamento anual da empresa $(\mathbf{R} \$)$ : } \\
\hline & \multicolumn{2}{|c|}{ Localização da empresa: } \\
\hline & \multirow{11}{*}{$\begin{array}{l}\text { Qual o setor de atuação da } \\
\text { empresa? }\end{array}$} & Derivados de carne \\
\hline & & Beneficiamento de café, chá e cereais \\
\hline & & Derivados de frutas e vegetais \\
\hline & & Derivados de trigo \\
\hline & & Desidratados e supergelados \\
\hline & & Óleos e gorduras \\
\hline & & Laticínios \\
\hline & & Açucares \\
\hline & & \begin{tabular}{|l|} 
Conservas de pescado \\
\end{tabular} \\
\hline & & Chocolate, cacau e balas \\
\hline & & Outros \\
\hline & \multirow{2}{*}{$\begin{array}{l}\text { Qual a origem da } \\
\text { empresa: }\end{array}$} & nacional \\
\hline & & multinacional \\
\hline & \multirow{2}{*}{ Possui filiais: } & Sim \\
\hline & & Nao \\
\hline & \multicolumn{2}{|c|}{ Número de instalações da empresa (matriz e filiais): } \\
\hline & \multicolumn{2}{|c|}{ Número de funcionários: } \\
\hline
\end{tabular}


Anexo A. Continuação...

\begin{tabular}{|c|c|c|c|}
\hline \multicolumn{4}{|c|}{$\begin{array}{l}\text { QUESTIONÁRIO DE PESQUISA: } \\
\text { A presente pesquisa acadêmica tem como objetivo aprofundar conhecimentos a respeito da previsão de } \\
\text { demanda nas indústrias de alimentos. }\end{array}$} \\
\hline \multirow{33}{*}{$\begin{array}{l}\text { PROCESSO DE } \\
\text { PREVISÃO DE } \\
\text { DEMANDA }\end{array}$} & \multicolumn{3}{|c|}{ Quantas pessoas participam da elaboração do processo de previsão de demanda? } \\
\hline & \multicolumn{3}{|c|}{ Qual o tempo gasto (em horas) no processo mensal de previsão de demanda? } \\
\hline & \multicolumn{3}{|c|}{ Qual o investimento mensal de recursos no processo de previsão de demanda? } \\
\hline & \multirow{2}{*}{$\begin{array}{l}\text { É utilizado algum modelo } \\
\text { ou método estatístico de } \\
\text { previsão de demanda? }\end{array}$} & \multicolumn{2}{|c|}{ Sim } \\
\hline & & \multicolumn{2}{|l|}{ Nao } \\
\hline & \multirow{12}{*}{ Qual o método utilizado ? } & \multirow{5}{*}{ Modelos de Julgamento: } & Simulação \\
\hline & & & Análise de mercado \\
\hline & & & Delphos \\
\hline & & & Painel de especialistas \\
\hline & & & Analogia Histórica \\
\hline & & \multirow{4}{*}{$\begin{array}{l}\text { Modelos de Séries } \\
\text { temporais }\end{array}$} & média móvel \\
\hline & & & alisamento exponencial \\
\hline & & & ARIMA \\
\hline & & & Decomposição \\
\hline & & \multirow{3}{*}{ Modelos de Causa e Efeito } & Regressão \\
\hline & & & Econométricos \\
\hline & & & Redes neurais \\
\hline & \multirow{2}{*}{$\begin{array}{l}\text { É utilizado algum } \\
\text { software para previsão de } \\
\text { demanda? }\end{array}$} & \multicolumn{2}{|l|}{ Sim } \\
\hline & & \multicolumn{2}{|l|}{ Nao } \\
\hline & \multirow{6}{*}{$\begin{array}{l}\text { Qual o horizonte de tempo } \\
\text { utilizado para previsão? }\end{array}$} & \multicolumn{2}{|l|}{ Mensal } \\
\hline & & \multicolumn{2}{|l|}{ Trimestral } \\
\hline & & Anual & \\
\hline & & Bimensal & \\
\hline & & Semestral & \\
\hline & & Bianual & \\
\hline & \multicolumn{3}{|c|}{ Quantos períodos são necessários de antecedência? } \\
\hline & \multicolumn{3}{|c|}{ Qual a freqüência para a realização das previsões? } \\
\hline & \multirow{5}{*}{$\begin{array}{l}\text { Quais as principais } \\
\text { dificuldades encontradas } \\
\text { no processo de previsão de } \\
\text { demanda? }\end{array}$} & \multicolumn{2}{|l|}{ Infra-estrutura } \\
\hline & & \multicolumn{2}{|c|}{ Capacitação e treinamento } \\
\hline & & \multicolumn{2}{|c|}{ Pouco comprometimento de outros colaboradores } \\
\hline & & \multicolumn{2}{|c|}{ Recursos Humanos } \\
\hline & & \multicolumn{2}{|l|}{ Disponibilidade de dados } \\
\hline & \multicolumn{3}{|c|}{ Qual a acurácia do processo de previsão de demanda em relação mês anterior? } \\
\hline
\end{tabular}

